\title{
Theory of anomalous magnetic interference pattern in mesoscopic superconducting/normal/superconducting Josephson junctions
}

\author{
Daniel E. Sheehy, Alexandre M. Zagoskin ${ }^{1,2}$ \\ ${ }^{1}$ Department of Physics and Astronomy, University of British Columbia, \\ 6224 Agricultural Road, Vancouver, B.C. V6T1Z1, Canada \\ ${ }^{2}$ D-Wave Systems Inc., 320-1985 W. Broadway, Vancouver, B.C., V6J 4Y3, Canada
}

(Dated: January 27, 2003)

\begin{abstract}
The magnetic interference pattern in mesoscopic SNS Josephson junctions is sensitive to the scattering in the normal part of the system. In this paper we investigate it, generalizing Ishii's formula for current-phase dependence to the case of normal scattering at NS boundaries in an SNS junction of finite width. The resulting flattening of the first diffraction peak is consistent with experimental data for S-2DEG-S mesoscopic junctions.
\end{abstract}

\section{INTRODUCTION}

The Fraunhofer diffraction pattern characterizing the dependence of the critical Josephson current on an applied external magnetic flux is a well-known feature of tunneling or dirty superconducting-normalsuperconducting (SNS) junctions ${ }^{1}$. The latter is defined by the condition that quasiparticle scattering length by impurities in the normal layer, $l_{i}$, is much less than the characteristic dimensions $(W, L)$ of this layer. For the case of clean SNS junctions, this pattern is more complex and demonstrates a distinct triangular central peak ${ }^{2}$. The pattern is $\Phi_{0}$-periodic in either case. More recent experimental and theoretical efforts have revealed, that in mesoscopic SNS junctions this pattern may be strongly altered by geometrical effects. For example, Heida et $\mathrm{a}^{\mathrm{l}^{3}}$ observed a $2 \Phi_{0}$-periodic diffraction pattern in narrow junctions, in contrast to the expected $\Phi_{0}$. The essential features of this behavior were explained theoretically ${ }^{4.5}$ using a semiclassical technique that accounts for the restricted geometry in terms of the classical trajectories associated with current-carrying Andreev states states in the normal layer.

In this paper we generalize this approach to include the effects of normal reflection at the NS boundaries, which are treated in the Blonder-Tinkham-Klapwijk (BTK) approximation ${ }^{6}$. The motivation for this work was provided by the JHAT (Jensen, Harada, Akazaki, and Takayanagi) experiment ${ }^{7}$. They observed critical current oscillations vs. flux in a wide ballistic S-2DEG-S junction, which exhibited apparently random small-scale (a fraction of $\Phi_{0}$ ) oscillations on top of a flattened first interference peak. As the first step towards understanding this data, here we extend previous work ${ }^{4.5}$ (which has focused mainly on the case without normal reflection at the NS boundary) by including closed trajectories formed by reflection from the walls of the device as well as from the NS boundaries. Such trajectories may be important because, for them, the effective area penetrated by the magnetic field (and therefore the field-dependent phase of the corresponding contribution to the supercurrent) change smoothly with their variations. By contrast, the contribution due to chaotic trajectories is expected to exhibit a rapid change with variation of the trajectory and thus the field dependence effectively cancels on scales below $\Phi_{0}$, like in an SNS junction in the diffusive limit. We show, that such an approach captures some, but not all, essential features of the experiment.

The general idea behind the quasiclassical approach is to represent the Josephson current through the SNS junction as a sum of contributions from various quasiclassical trajectories linking the superconductors $\stackrel{4}{ }$. Such a generalization of Ishii's formula ${ }^{8}$ follows from the quasiclassical Eilenberger equations and gives a transparent description suitable for complicated geometries. We will assume that the conditions of its applicability $\left(\lambda_{F} \ll \xi_{0} \ll W, L\right.$, where $\lambda_{F}$ is the Fermi wavelength and $\xi_{0}$ is the superconducting coherence length ["Cooper pair size"]) are satisfied. Our approach is to first calculate the density $N(\xi)$ of Andreev states in the normal area and then express the Josephson current through it. (Here $\xi$ is the quasiparticle energy measured from the Fermi level.) For simplicity, we consider the case of a rectangular normal region with dimensions $W \times L$ (see Fig. (1), with specular normal reflections from the boundaries (given by the line segments $C D$ and $F E$ in Fig. (1).

The flux $\Phi=H W L$ produced by the magnetic field $\vec{H}=H \vec{e}_{z}$ through the normal region can be described by the vector potential

$$
\vec{A}=-H y \cdot \vec{e}_{x}
$$

The applied magnetic field is screened by the superconducting regions, producing screening currents over a length given by $\lambda$, the magnetic penetration depth. As is standard $\underline{\underline{1}}$, we shall neglect the variation of the superconducting phase and magnetic field over this length scale; thus, our results apply at $W, L \gg \lambda$.

Before turning to the calculation of the current, let us briefly remark on why closed trajectories are of principal importance. The introduction of normal reflection on NS boundary takes away the simplicity of quasiclassical trajectories with Andreev reflections only (when the hole retraces the electron's path). Even if assume (as we do), that the Andreev reflection exactly reverts the group velocity of a quasiparticle, and that a normal reflection is specular, the quasiparticle trajectory will be generally 


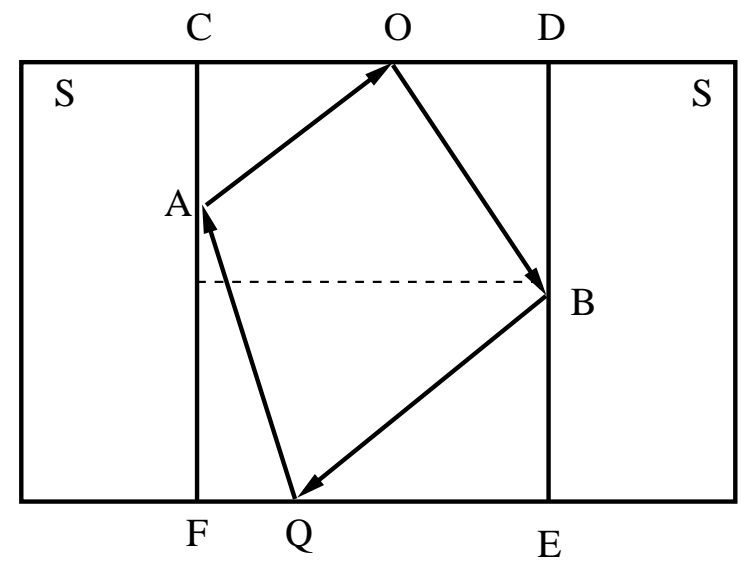

FIG. 1: Schematic picture of a closed quasiparticle trajectory carrying supercurrent between the superconducting leads (labelled $\mathrm{S}$ ). The dashed line is at $y=0$.

open, with multiple reflection points at an NS boundary. Each SNS leg of such a trajectory coherently contributes to the latter's partial Josephson current $I_{t r}$, with wildly varying phase factors. Therefore the total contribution of such trajectories will be suppressed. The exceptions are closed trajectories, where phase gains are systematic. Such trajectories contribute most to the quasiclassical density of states ${ }^{9}$. If normal reflection amplitude from NS boundary is small, $|\mathcal{R}| \ll 1$, the leading contribution will be from the trajectories with the minimum possible number of reflections on the NS boundaries (one each, see Fig. (1) (class A trajectories), which simplifies the situation. On the other hand, in this case we can not discard the contributions from open (class B) trajectories, since only the first leg will significantly contribute to the current, and the above "phase cancelling" argument no longer applies.

In the following, we assume that 1) Andreev reflection on NS boundary prevails and 2) the scattering in the normal region is negligible ${ }^{10}$. We will therefore concentrate on the two above classes of trajectories. Consider then the closed trajectory AOBQ in Fig. 1 where electrons propagate clockwise, and holes, related to the electrons by Andreev reflections from NS boundaries (at A and B in Fig. 1), propagate counterclockwise. (The results presented here are valid for a trajectory with an arbitrary number of reflections from the side walls). The phase gain for an electron from the magnetic field along $\mathrm{AOB}$ is simply related to the flux enclosed by the trajectory and the dashed line $y=0$. We introduce the phase gains $w$ and $\bar{w}$ along $\mathrm{AOB}$ and BQA, via

$$
\begin{aligned}
w \cdot \Phi_{0} & =\int_{A O B} \vec{A} \cdot d \vec{l}=-H \int_{0}^{L} y(x) d x, \\
& =-H\left(S_{A O B E F}-W L / 2\right) \\
\bar{w} \cdot \Phi_{0} & =\int_{B Q A} \vec{A} \cdot d \vec{l}=-H \int_{L}^{0} y(x) d x, \\
& =+H\left(S_{B Q A F E}-W L / 2\right) .
\end{aligned}
$$

We have expressed the appropriate area in Eq. (2) [and similarly in Eq. (3)] as the difference of the area below AOB (which we denote by $S_{A O B E F}$ ) and the area below the dashed line. Our expression for the Josephson current carried by a particular trajectory will involve both the sum, $\nu \equiv w+\bar{w}$, and difference, $\Omega \equiv w-\bar{w}$, of these phases:

$$
\begin{aligned}
\nu \cdot \Phi_{0} & =-H\left(S_{A O B E F}-S_{B Q A F E}\right) \\
& =-H S_{A O B Q} \equiv-\Phi_{e x} \\
\Omega \cdot \Phi_{0} & =-H\left(S_{A O B E F}+S_{B Q A F E}\right)+H W L
\end{aligned}
$$

where $\Phi_{e x}$ is the flux of the external field through the loop $S_{A O B Q}$. Let us compute the phase gains (due to the field and from propagation) accumulated by quasiparticles travelling along $A O B$ and $A Q B$. Denoting the electron (hole) momentum at energy $E$ by $k(q)$, and setting $|A O B|=l,|B Q A|=\bar{l}$, we can write for the electron (hole) wave function the following expressions for the phase gains along trajectories:

$$
\begin{aligned}
& \psi_{e}(A) \stackrel{A O B}{\leftrightarrows} \psi_{e}(A) e^{i(k l+\pi w)} \\
& \psi_{h}(A) \stackrel{A O B}{\leftrightarrows} \psi_{h}(A) e^{i(q l-\pi w)} \\
& \psi_{e}(B) e^{i(k \bar{l}+\pi \bar{w})} \stackrel{A Q B}{\leftrightarrows} \psi_{e}(B) \\
& \psi_{h}(B) e^{i(q \bar{l}-\pi \bar{w})} \stackrel{A Q B}{\leftrightarrows} \psi_{h}(B)
\end{aligned}
$$

Due to the proximity effect, the quasiparticle wave functions in the normal region are composed of electron and hole components, which are mixed by Andreev reflections on NS boundaries. Thus, we have

$$
\begin{aligned}
& \Psi_{A O B}(x)=a \psi_{e} \Longleftrightarrow(x)+b \psi_{h} \Longleftarrow(x), \\
& \Psi_{B Q A}(x)=\bar{a} \psi_{e}^{\Longleftarrow}(x)+\bar{b} \psi_{h}^{\Longleftrightarrow}(x) .
\end{aligned}
$$

Here, the arrows indicate the direction of the group velocity projection on the x-axis. The amplitudes of normal and Andreev reflection are denoted by $\mathcal{R}$ and $\mathcal{A}=-i|\mathcal{A}| e^{ \pm i \chi}$, respectively.

For the phase of the latter we have taken the result that is valid for a clean NS boundary and for a quasiparticle exactly on the Fermi surface $\left(\xi \equiv E-E_{F}=0\right)$. The unitarity conditions, which encode the notion that, e.g., an electron propagating along $B Q A$ must undergo either 
Andreev or normal reflection at $A$, can be written as:

$$
\begin{gathered}
\bar{a} \psi_{e}(B) e^{i(k \bar{l}+\pi \bar{w})}=\mathcal{R} a \psi_{e}(A)-i|\mathcal{A}| e^{-i \chi_{A}} \bar{b} \psi_{h}(B) e^{i(q \bar{l}-\pi \bar{w})}, \\
b \psi_{h}(A)=\mathcal{R} \bar{b} \psi_{h}(B) e^{i(q \bar{l}-\pi \bar{w})}-i|\mathcal{A}| e^{i \chi_{A}} a \psi_{e}(A), \\
a \psi_{e}(A) e^{i(k l+\pi w)}=\mathcal{R} \bar{a} \psi_{e}(B)-i|\mathcal{A}| e^{-i \chi_{B}} b \psi_{h}(A) e^{i(q l-\pi w)}, \\
\bar{b} \psi_{h}(B)=\mathcal{R} b \psi_{h}(A) e^{i(q l-\pi w)}-i|\mathcal{A}| e^{i \chi_{B}} \bar{a} \psi_{e}(B) .
\end{gathered}
$$

In the next section, we shall use Eq. (10) to find the density of Josephson current-carrying levels, which will yield the Josephson current ${ }^{11}$.

\section{DISPERSION LAW}

In the above system of equations, we can (up to normalization and immaterial phase factors) assume $\psi_{e}(A)=\psi_{h}(A)=\psi_{e}(B)=\psi_{h}(B) \simeq 1$, which leaves us with a homogeneous system of linear equations for the quantities $\{a, \bar{a}, b, \bar{b}\}$, with matrix

$$
M=\left[\begin{array}{cccc}
\mathcal{R} & -e^{i(k \bar{l}+\pi \bar{w})} & 0 & -i|\mathcal{A}| e^{-i \chi_{A}} e^{i(q \bar{l}-\pi \bar{w})} \\
-i|\mathcal{A}| e^{i \chi_{A}} & 0 & -1 & \mathcal{R} e^{i(q \bar{l}-\pi \bar{w})} \\
-e^{i(k l+\pi w)} & \mathcal{R} & -i|\mathcal{A}| e^{-i \chi_{B}} e^{i(q l-\pi w)} & 0 \\
0 & -i|\mathcal{A}| e^{i \chi_{B}} & \mathcal{R} e^{i(q l-\pi w)} & -1
\end{array}\right]
$$

The dispersion law for the supercurrent-carrying quasiparticles follows from the solvability condition, $\operatorname{det} M=$ 0 . Henceforth, we explicitly take $l=\bar{l}$, which is true in case of specular normal reflection and a rectangular normal region. Introducing $\chi \equiv \chi_{B}-\chi_{A}$, we have

$$
\begin{aligned}
\operatorname{det} M= & 2 \mathrm{e}^{i(k+q) l} \cdot D, \\
D \equiv \cos ( & \nu \pi+l(k-q))+|\mathcal{A}|^{2} \cos (\chi-\Omega \pi) \\
& -|\mathcal{R}|^{2} \cos (k+q) l .
\end{aligned}
$$

At this point we proceed by making an assumption about the energy-dependent amplitudes for Andreev and normal reflection. As we are interested in the possibility of normal reflection at the interface, we take these from the results of BTK. Thus, at low energies, we have ${ }^{6}$

$$
\begin{aligned}
\mathcal{A} & =\frac{-i \Delta \mathrm{e}^{-i \chi}}{2 Z \sqrt{\Delta^{2}-E^{2}}} \\
\mathcal{R} & =\sqrt{1-|\mathcal{A}|^{2}}
\end{aligned}
$$

The momentum of an electron (hole) with energy $\xi$ is $k( \pm \xi)$; thus we introduce the dimensionless quantity $\varphi(\xi)=k(\xi) \mathcal{L} / 2=\sqrt{2 m\left(E_{F}+\xi\right)} \cdot l$ and define $\phi_{ \pm}(\xi) \equiv \varphi(\xi) \pm \varphi(-\xi)$, giving

$$
\begin{gathered}
D(\xi, \nu)=\cos \left(\phi_{-}+\pi \nu\right)+\frac{\Delta^{2}}{4 Z^{2}\left(\Delta^{2}-\xi^{2}\right)} \cos (\chi-\pi \Omega) \\
+\frac{\Delta^{2}}{4 Z^{2}\left(\Delta^{2}-\xi^{2}\right)} \cos \phi_{+}-\cos \phi_{+}
\end{gathered}
$$

In order to calculate the density of states, we will use the approach due to Slutskin ${ }^{12}$ (applied to SNS systems in Ref. 13) to extract the density of current-carrying states $N(\xi)$ directly from the dispersion law. Thus, $N(\xi)=\sum_{s} \delta\left(\xi-\xi_{s}\right)$, where $\xi_{s}$ are given by the solutions of dispersion equation $D(\xi, \nu)=0$. A simple variable change yields

$$
\begin{aligned}
& N(\xi)=\left|\frac{\partial D(\xi, \nu)}{\partial \xi}\right| \delta(D(\xi, \nu)) \\
& \frac{\partial D(\xi, \nu)}{\partial \xi} \approx-\frac{2 l}{v_{\mathrm{F}}} \sin \left(\phi_{-}+\pi \nu\right),
\end{aligned}
$$

where in Eq. (18) we have displayed the low-energy limit of $\partial D(\xi, \nu) / \partial \xi$ and approximated $\partial \phi_{-} / \partial \xi \sim 2 l / v_{\mathrm{F}}$ and $\partial \phi_{+} / \partial \xi \sim 0$

The delta-function constraint in Eq. (17) implies that

$$
\begin{aligned}
\phi_{-} & +\pi \nu+(2 m+1) \pi \\
= & \pm \arccos \left(\frac{\Delta^{2}}{4 Z^{2}\left(\Delta^{2}-\xi^{2}\right)}[\cos (\chi-\pi \Omega)+1]-1\right)
\end{aligned}
$$

with $m$ being an integer. Let us briefly pause to discuss (qualitatively) the physical meaning of Eq. (19). The argument of the arccos in Eq. (19) has the interpretation of a generalized phase difference across the junction. Since the current is given by differentiating the free energy with respect to the phase difference ${ }^{11}$, we see that the \pm in Eq. (19) correspond to levels that carry current in opposite directions. We shall refer to these two distinct contributions to $N(\xi)$ as $N_{ \pm}(\xi)$. The prefactor $|\partial D / \partial \xi| \approx \frac{2 l}{v_{\mathrm{F}}}\left|\sin \left(\phi_{-}+\pi \nu\right)\right|$ assigns a particular weight to the $\delta$-function contributions to $N_{ \pm}$; a direct application of the Poisson summation formula 14 yields (taking the low-energy limit)

$$
\begin{aligned}
N(\xi) & =N_{+}(\xi)+N_{-}(\xi) \\
N_{ \pm}(\xi) & \equiv \sum_{n=-\infty}^{\infty}(-1)^{n} \frac{l}{\pi v_{\mathrm{F}}} e^{i \pi \nu n} e^{i n \phi_{-}(\xi) \mp i n \Gamma(\chi-\pi \Omega)} \\
\Gamma(\chi) & \equiv \arccos \left(\frac{1}{4 Z^{2}}[\cos \chi+1]-1\right)
\end{aligned}
$$

where we have have rescaled the barrier height $Z$ via $Z \rightarrow Z \Delta$ and we choose a branch of the arccos in Eq. (21) 
such that $\Gamma$ smoothly goes to $\chi-\pi \Omega$ for $Z \rightarrow 1 / 2$. In the next section, we apply Eq. (21) to the calculation of the current due to a single trajectory, which (as we have briefly noted above) is related to the difference $N_{+}(\xi)-$ $N_{-}(\xi)$.

\section{JOSEPHSON CURRENT DUE TO A PARTICULAR TRAJECTORY}

Having determined the density of states $N(\xi)$ associated with a particular trajectory, we now turn to the computation of the associated current. In the limit of zero normal reflectance the two terms $N_{ \pm}(\xi)$ in Eq. (21) correspond to two separate groups of Andreev levels (AOB and BQA) which carry current in opposite directions and also move in opposite directions as $\chi$ is changed ${ }^{15}$. The simplicity of this expression is deceptive: the length $l$ and phase gains $\nu, \Omega$ depend nontrivially on the shape of the trajectory. The associated contribution to the Josephson current is given in terms of $N_{ \pm}(\xi)$ as 11

$$
I_{\mathrm{tr} .}=\frac{-e}{l} \int_{-\infty}^{\infty} d \xi \tanh \left(\frac{\beta \xi}{2}\right)\left(N_{+}(\xi)-N_{-}(\xi)\right) v_{F x},
$$

where $e$ is the electric charge; henceforth we shall set $e=1$. In Eq. (22), the subscript "tr." denotes that this is the contribution due to one particular trajectory, such as that pictured in Fig. 11. At low energies, $\phi_{-}(\xi) \approx 2 \xi l / v_{\mathrm{F}}$. Utilizing the integral formula

$$
\int_{-\infty}^{\infty} d \xi \tanh \left(\frac{\beta \xi}{2}\right) e^{i n 2 \xi l / v_{\mathrm{F}}}=\frac{2 \pi i\left(1-\delta_{n, 0}\right)}{\beta \sinh \left(2 \pi \ln /\left(\beta v_{\mathrm{F}}\right)\right)},
$$

we find for the current

$$
I_{\mathrm{tr} .}=\sum_{n=1}^{\infty} \frac{8 \cos (\pi \nu n) v_{F x}}{\beta v_{\mathrm{F}} \sinh \left(2 \pi l n /\left(\beta v_{\mathrm{F}}\right)\right)}(-1)^{n+1} \sin n \Gamma(\chi-\pi \Omega) .
$$

Equation (23), together with Eqs.(20), (21), is the central result of this paper ${ }^{16}$.

For closed-loop trajectories we must take into account that there always will be a related contribution from the same trajectory, but in the counterclockwise direction (we completely neglect the dynamical effects of the magnetic field). In the next section, we will consider the dominant classes of trajectory which contribute to the current. Before doing so, however, we pause to note that in the limit $\nu \rightarrow 0, Z \rightarrow 1 / 2$, Eq. 23) reduces to the Ishii's formula ${ }^{8}$ for a wide clean SNS junction,

$$
I_{\mathrm{tr} .}^{(0)}(\chi)=\sum_{n=1}^{\infty} \frac{8 v_{F x}}{\beta v_{\mathrm{F}} \sinh \left(2 \pi l n /\left(\beta v_{\mathrm{F}}\right)\right)}(-1)^{n+1} \sin n \chi .
$$

Despite this similarity, our expression Eq. (23) generalizes Eq. (24) by incorporating normal reflection. In particular, our expression is not expected to yield a $2 \Phi_{0^{-}}$ periodic pattern seen in the theoretical results $\frac{4}{2}, \frac{5}{3}$ because it does not include, e.g., non-horizontal straight trajectories, unless they are a part of a (closed) class A trajectory.

\section{SUMMATION OVER CLASSES OF TRAJECTORIES}

In the present section we apply the results of the previous section to calculate the contribution to the Josephson current due to various types of quasiparticle trajectories. We assume that each such contribution has the form of the general expression Eq. (23) but with values of $\nu, l$ and $\Omega$ taken from the geometrical properties of the associated trajectory.

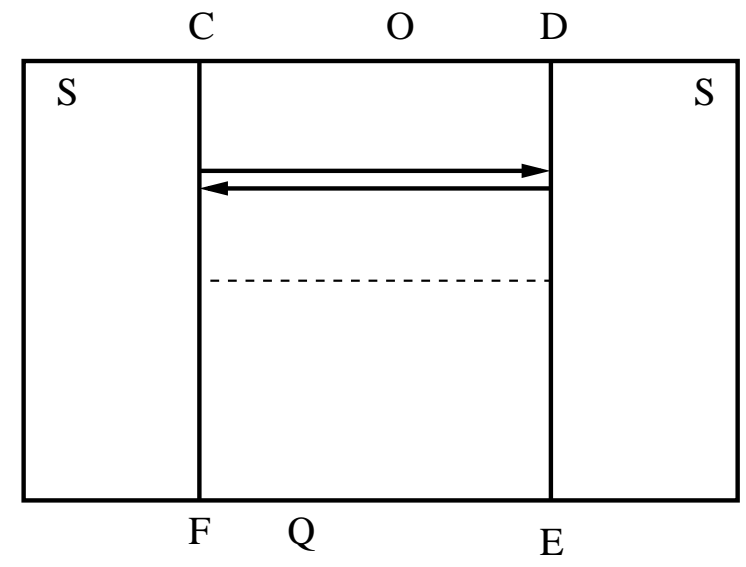

FIG. 2: Typical straight trajectory contributing to the Josephson current. The intercept of the trajectory along the segment $C F$ is the coordinate $y$ in Eq. 25).

We begin by discussing the leading-order contribution due to straight trajectories, which undergo no reflections from the sides of the device. Due to the fact that normal reflection is a specular process, the trajectories must have incoming angle $\theta=0$, as depicted in Fig. [2 It is easily seen that for them, the phase gains [i.e. Eqs. (46)] due to the magnetic field are $\nu=0$ and $\Omega=-2 y \phi / W$, where $\phi \equiv \Phi / \Phi_{0}$ with $\Phi_{0}$ the flux quantum. The length $l$ is given by $L$, and $v_{F x}=v_{\mathrm{F}}$. Such trajectories are labeled by the coordinate $y$, given by their intercept on the line segment $C F$ in Fig. 2 To incorporate all such trajectories, we integrate over $y$ from $-W / 2$ to $W / 2$, dividing by an overall normalization factor $W$. Thus, we obtain for the current $I_{\mathrm{s}}$ due to the straight trajectories

$$
I_{\mathrm{S}} \approx \frac{4 v_{\mathrm{F}} x}{L \pi} \int_{-\frac{1}{2}}^{\frac{1}{2}} d y \sum_{n=1}^{\infty} \frac{(-1)^{n+1}}{\sinh n x} \sin n \Gamma(\chi-2 y \phi),
$$

where for simplicity we have rescaled the dimensional coordinate $y \rightarrow W y$ to make it dimensionless and have defined $w \equiv W / L$ to be the dimensionless width of our sample. The parameter $x \equiv 2 \pi L / v_{\mathrm{F}} \beta$ is a dimensionless measure of the temperature. 


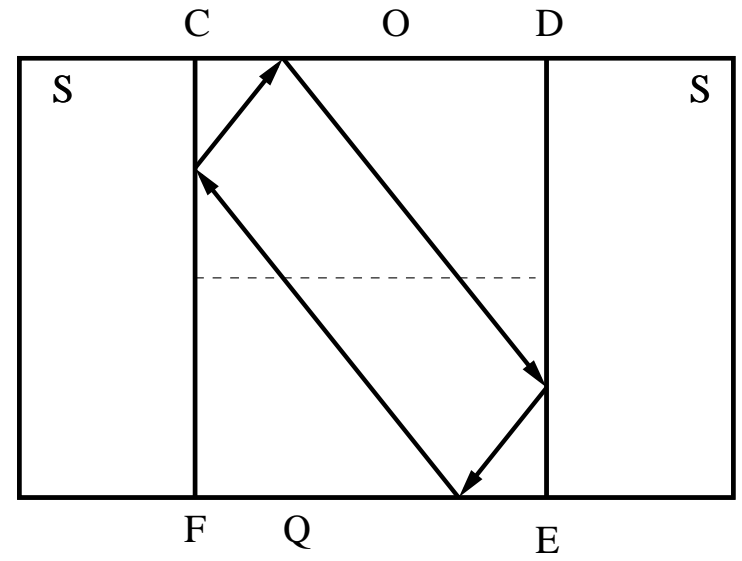

FIG. 3: A typical bouncing trajectory. The intercept of the trajectory on the line segment $C F$ is the coordinate $y$ in Eq. (29).

Now consider the contribution due to class A (closed) bouncing trajectories, which bounce specularly once from the walls of the device. The requirement that the trajectory bounce specularly means, that there is only one for each intercept $y$. To compute this contribution, we must calculate the values of $l, \nu$ and $\Omega$. These are found geometrically to be

$$
\begin{aligned}
l & =L \sqrt{1+w^{2}} \\
\nu & =2 \phi\left(y^{2}-\frac{1}{4}\right) \\
\Omega & =0,
\end{aligned}
$$

where we have again rescaled $y \rightarrow y W$. Summing over all such trajectories amounts to integrating over $y$ (and normalizing by an overall factor of $1 / W$ as before) and multiplying by an overall factor of 2 to include counterclockwise trajectories. Thus, we find for the current $I_{\mathrm{b}}$ due to the bouncing trajectories:

$$
\begin{gathered}
I_{\mathrm{b}} \approx \frac{8 v_{\mathrm{F}} x}{\pi L \sqrt{1+w^{2}}} \int_{-\frac{1}{2}}^{\frac{1}{2}} d y \sum_{n=1}^{\infty} \frac{\cos (\pi \nu n)}{\sinh n x \sqrt{1+w^{2}}} \\
\quad \times(-1)^{n+1} \sin n \Gamma(\chi) .
\end{gathered}
$$

The generalization of Eq. (29) to the case of multiple reflections from the sides of the device is straighforward.

\section{RESULTS AND DISCUSSION}

Having noted the various dominant contributions to the current, we now turn to the calculation of the critical Josephson current $I_{\mathrm{c}}$ as a function of the applied flux. This quantity is defined in terms of the total Josephson current $I\left(=I_{\mathrm{s}}+I_{\mathrm{b}}\right)$ as

$$
\left.I_{\mathrm{c}}(\phi) \equiv \max \{I(\chi, \phi)\}\right|_{0 \leq \chi<2 \pi}
$$

We begin by noting that the contribution due to the bouncing trajectories (i.e, Eq. (29)) is explicitly dependent on the width-to-length ratio of the junction $w$ and indeed vanishes for large $w$. Thus, we can effectively isolate the effect of the straight trajectories by examining a very wide sample. In Fig. 4 we display numerical calculations of $I_{c}(\phi)$ for the case $w=50, Z=1,2,10$, and $x=0$ (i.e., $T=0$ ). (All curves have been normalized to their values at $\phi=0$.)

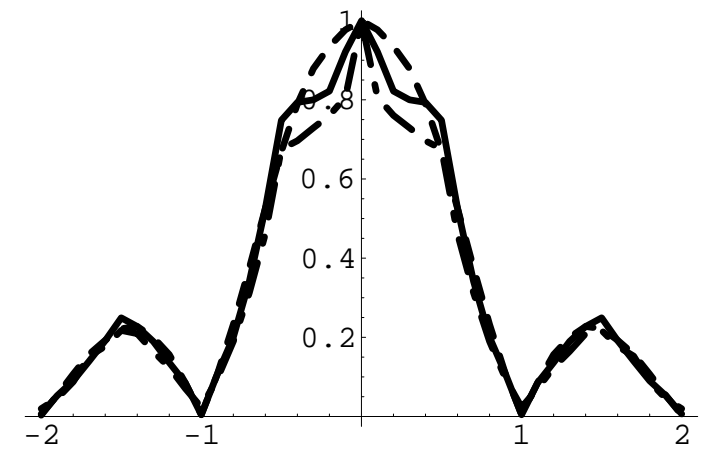

FIG. 4: The evolution of the normalized critical current (computed numerically) as a function of the external flux $\Phi / \Phi_{0}$ for the case of a wide junction. The dot-dashed curve is $Z=1$, the solid curve is $Z=2$ and the dashed curve is $Z=10$.

The pattern in Fig. 4 is $\Phi_{0}$-periodic (i.e., $I_{\mathrm{c}}$ first vanishes at $\phi= \pm 1$ ), which is expected for a wide junction 4 . For a barrier height on the order of the pair potential ( $Z=1$, dot-dashed curve), there is clearly a flattening of $I_{\mathrm{c}}$ below $\phi= \pm 1$ arising from the energy-dependent amplitude for Andreev reflection. This flattening (but not the sharp peak for $\phi \simeq 0$ ) is reminiscent of the flattening effect seen in the JHAT experiment ${ }^{7}$. This effect disappears for larger barrier heights, as exhibited by the $Z=10$ curve (dashed line) in Fig. 4. This shows approximate reverting to the Fraunhofer shape is to be expected, as the contribution of higher-order processes to the Josephson current is suppressed by increased normal reflection probability. At smaller values of $w$, the increased importance of the bouncing trajectories leads to somewhat different behavior of the $I_{\mathrm{c}}(\phi)$ curve as a function of $Z$. In Fig. 5] we display $I_{\mathrm{c}}(\phi)$ for the case of a narrow junction (i.e., $w=1$ ) for the cases $Z=1,2,10$ and $|\phi|<1$. This curve is also for the case of $T=x=0$. The $Z=1$ curve (dashed line) does exhibit flattening for small values of $\phi$. In contrast to the wide junctions, increasing the barrier height leads to a sharpening of the $I_{\mathrm{c}}$ curve near $\phi=0$, as seen in the $Z=10$ curve (dash-dot line).

\section{CONCLUDING REMARKS}

In this paper we have studied the effects of a finite tunneling barriers and restricted geometries on the Joseph- 


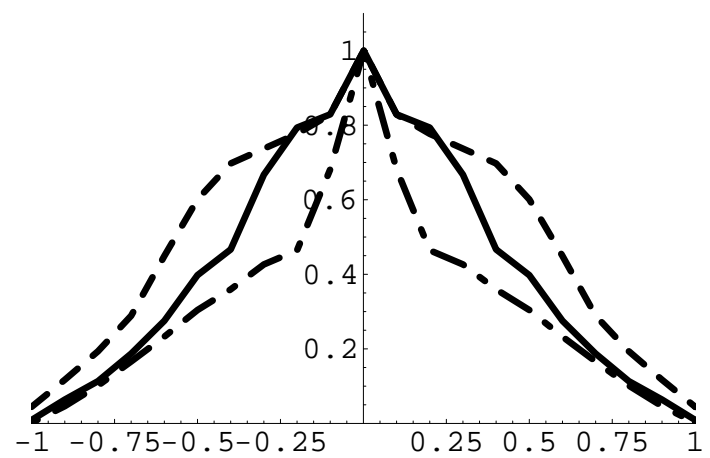

FIG. 5: The evolution of the normalized critical current (computed numerically) as a function of the external flux $\Phi / \Phi_{0}$ for the case of a narrow junction $(w=1)$, indicating a progressive sharpening of the Fraunhofer diffraction pattern as $Z$ is increased. The dot-dashed curve is $Z=10$, the solid curve is $Z=2$ and the dashed curve is $Z=1$.

son effect in a clean junction. We derived closed formulas for the density of states and Josephson current in the system, which generalize the known results to the case of BTK normal scattering on NS interfaces, which allow an intuitive insight in the mechanisms of non-Fraunhofer diffraction patterns in mesoscopic SNS junctions and can be used in complex geometries.

As an application of the technique, we have calculated the critical Josephson current $I_{\mathrm{c}}$ in a rectangular SNS junction for various values of the junction width and the tunneling barrier height $Z$. As expected, the deviation of $I_{\mathrm{c}}(\phi)$ from the case of a clean wide junction is most pronounced at small $\phi$, leading to a flattening effect, reminiscent of the experimental results of JHAT ${ }^{7}$. Nevertheless there remain open questions.

First, our calculations do not provide a quantitative match. More importantly, they do not reproduce the fine oscillations superimposed on the flattened first peak, seen in the experiment of Ref. 7. A possible source of this effect can be due to the closed trajectories with multiple reflection points, not taken into account here. The fine structure in the density of states in SN billiard structures (i.e., so-called Andreev billiards) arises from such trajectories ${ }^{9}$, in accordance with the Gutzwiller formula, which make this scenario at least plausible. Finally, we neglected the elastic scattering inside the normal region, which was significant in Ref. 7. Generally, it would keep $l \neq \bar{l}$, so that that calculations up to Eq. (11) would hold, but the following formulas would be modified.

Acknowledgments: We gratefully acknowledge stimulating and enjoyable discussions with M.H.S.Amin, A.Golubov, Y.Harada, A.Maassen van den Brink, and H.Takayanagi. AZ thanks J.P. Hilton for valuable comments on the manuscript. This work was supported by NSERC.
1 See, e.g., M. Tinkham, Introduction to Superconductivity, McGraw-Hill, 1996.

2 A. V. Svidzinskii, Spatially inhomogeneous problems in the theory of superconductivity., Moscow (1982).

3 J. P. Heida, B. J. van Wees, T. M. Klapwijk and G. Borghs, Phys. Rev. B 57, R5618 (1998).

4 V. Barzykin and A. M. Zagoskin, Superlat. and Microstruct. 25, 797 (1999).

${ }^{5}$ U. Ledermann, A. L. Fauchère and G. Blatter, Phys. Rev. B 59, R9027 (1999).

${ }^{6}$ G. E. Blonder, M. Tinkham and T. M. Klapwijk, Phys. Rev. B 25, 4515 (1982).

7 S. Jensen, Y. Harada, T. Akazaki, and H. Takayanagi, "Anomalous Behaviour of the Critical Current in High Performance Nb/n-InAs/Nb SNS Junctions", ISEC'01, p.137 (2001).

8 G. Ishii, Progr. Theor. Phys. 44, 1525 (1970).

${ }^{9}$ I. Adagideli and P. Goldbart, Phys. Rev. B 65, 201306 (2002); Int. J. Mod. Phys. B 16, 1381 (2002).

10 The second assumption is generally justified in S-2DEG-S structures, where the elastic scattering length $l_{e}$ can exceed $1 \mu \mathrm{m}^{3}$, but does not hold in the JHAT experiment ${ }^{7}$, where $l_{e}=.2 \mu \mathrm{m}$ is on the order of the length of the normal region $(0.22$ to $0.72 \mu \mathrm{m})$, but not its width $(4.2 \mu \mathrm{m})$.

11 A. M. Zagoskin, Quantum Theory of Many-Body Systems,
Springer, 1998.

12 A.A. Slutskin, Zh. Eksp. Teor. Fiz. 58,1098 (1970) (Sov. Phys. JETP, 31, 589 (1970)).

13 H.A. Blom, A. Kadigrobov, A.M. Zagoskin, R.I. Shekhter and M. Jonson Phys. Rev. B 57, 9995 (1998).

14 i.e., $\sum_{m=-\infty}^{\infty} \delta(2 \pi \mu+2 \pi m)=\frac{1}{2 \pi} \sum_{n=-\infty}^{\infty} \mathrm{e}^{2 i \pi \mu n}$.

15 J. Bardeen and J.L. Johnson, Phys. Rev. B 5, 72 (1972).

16 This expression (23) is gauge-invariant. Indeed, under the gauge symmetry

$$
\begin{aligned}
& \mathbf{A}(\mathbf{r}) \rightarrow \mathbf{A}(\mathbf{r})+\frac{\Phi_{0}}{\pi} \nabla \varphi(\mathbf{r}), \\
& \chi(\mathbf{r}) \rightarrow \chi(\mathbf{r})-2 \varphi(\mathbf{r})
\end{aligned}
$$

the quantities $w$ and $\bar{w}$ transform as

$$
\begin{aligned}
& w \rightarrow w+(\varphi(B)-\varphi(A)) / \pi \\
& \bar{w} \rightarrow \bar{w}+(\varphi(A)-\varphi(B)) / \pi .
\end{aligned}
$$

Therefore, the argument of $\Gamma$ in Eq. (23) remains invariant. The quantity $\nu$ is also invariant, it being related to the flux through a closed loop [as noted in Eq. (5)]. Note that although subsequent equations [e.g., Eq. 25) and Eq. 29] may appear to be non gauge-invariant, this is because of the particular gauge choice Eq. (1). 